\title{
Analysis of Vehicle Theft: A Case Study in Malaysia using Functional Forms of Negative Binomial Regression Models
}

\author{
Malina Zulkifli ${ }^{1}$, Noriszura Ismail ${ }^{2}$ and Ahmad Mahir Razali ${ }^{3}$ \\ ${ }^{1}$ School of Quantitative Sciences, Universiti Utara Malaysia, MALAYSIA \\ ${ }^{2}$ School of Mathematical Sciences, Universiti Kebangsaan Malaysia, MALAYSIA \\ ${ }^{3}$ School of Mathematical Sciences, Universiti Kebangsaan Malaysia, MALAYSIA
}

Received: 7 Oct. 2012, Revised: 21 Dec. 2012, Accepted: 23 Dec. 2012

Published online: 1 Jun. 2013

\begin{abstract}
This paper proposes the application of the two well known models of negative binomial regression, namely the NB-1 and the NB-2, and the functional form of negative binomial regression, namely the NB-P, for the analysis of vehicle theft crime. The advantage of using the NB-P is that it parametrically nests both the NB-1 and the NB-2, and allows statistical tests of the NB-1 and the NB-2 models against a more general alternative. In this study, the functional forms of negative binomial regression model were fitted to vehicle theft crime data obtained and compiled from ten insurance companies in Malaysia. The results of this study indicate that the risks of vehicle theft are higher for vehicles above eight years, vehicles above 1800 c.c., local vehicles and vehicles located in the central area of Malaysia.
\end{abstract}

Keywords: crime index, vehicle theft, Poisson regression, negative binomial regression, functional form; overdispersion

\section{Introduction}

In Malaysia, the Royal Malaysia Police (RMP) uses 'crime index' to quantify crime. Crime index, which can be used as an indicator of the nature and the extent of crime and as a yardstick to evaluate the effectiveness of crime prevention measures, can be divided into two major types; violent and property crimes. In particular, violent crimes are crimes of violent that are sufficiently regular and significant in occurrence such as murder, robbery, rape and voluntarily causing hurt, whereas property crimes are offences of property loss where there are no use of violence by the perpetrators such as housebreaking and theft, theft of vehicle and other forms of theft. Even though violent crimes attract the greatest attention of both public and media in Malaysia, property crimes account for about ninety percents of all crimes reported throughout the thirty years of 1980-2009 [5]. Specifically, vehicle thefts, which are thefts of cars, lorries and motorcycles, contributes the majority of property crime, and the ratio of vehicle thefts to the overall thefts has been consistent throughout the years. As examples, in 2004, forty-nine percents of the total crime index reported were contributed by vehicle thefts [4], whereas in 2008 and 2009 , forty-two percent $(88,820$ out of 211,645 cases $)$ and thirty-eight percents $(80,662$ out of 211,184 cases $)$ of the total index crime were contributed by vehicle thefts [9].

Figure 1 shows the number of private car, motorcycle and total vehicle thefts in 2003-2010 based on theft claims reported to insurance companies in Malaysia. It is interesting to note that the number of motorcycle theft which escalates drastically in year 2006, and the number of private car theft which increases significantly in year 2008 , have sizeable effects on the total number of vehicle theft. In other words, the total number of vehicle theft increases quite significantly in these two particular years.

\footnotetext{
*Corresponding author e-mail: ni@ukm.my
} 


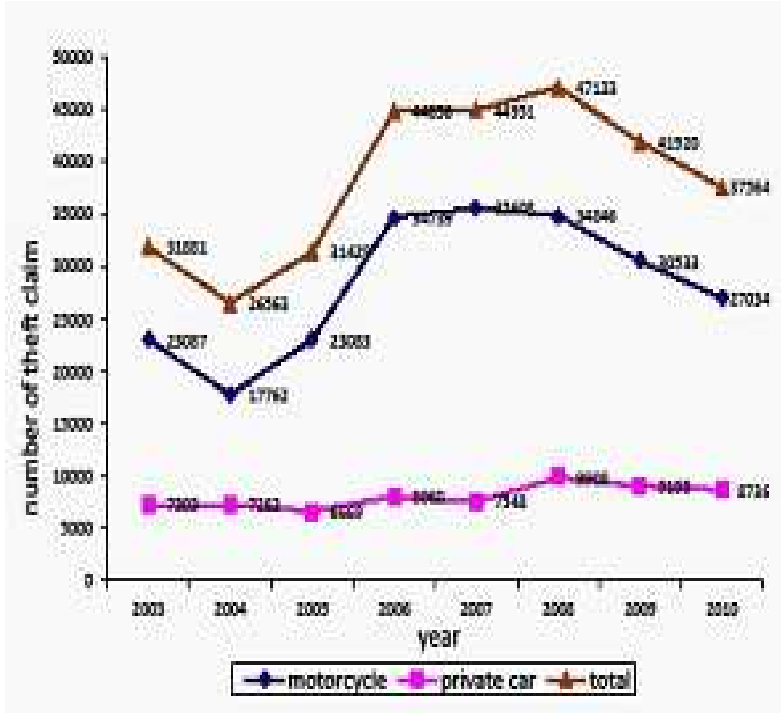

Fig. 1: Insurance industry statistics on stolen vehicles in Malaysia (2003-2010) Source: General Insurance Association of Malaysia

In criminological research, the least squares, the Poisson, the quasi Poisson and the negative binomial regression models have been proposed and applied for analyzing the relationship between crime and social, economic and demographic factors. As examples, [14] studied the relationship between inequality and crime using quasi Poisson regression model, [7] studied the effects of unemployment on property crime by fitting Poisson and negative binomial regression models, [20] estimated the effect of police presence on car theft by implementing least squares regression model, and [6] examined the effects of local inequality on property and violent crime in South Africa using negative binomial regression model. Based on insurance studies, Poisson regression model has been used for fitting claim data, and such examples can be found in [13] and [3] who fitted the model to datasets of U.K. motor insurance claim. However, insurance claims often display overdispersion and inappropriate imposition of Poisson regression model may underestimate the standard errors and overstate the significance of regression parameters. To account for overdispersion, quasi Poisson regression model has been applied. Such examples can be found in [17] who used the data provided by Lloyd's Register of Shipping and applied quasi Poisson regression model to damage incidents caused to the forward section of cargocarrying vessels to allow for possible inter-ship variability in accident proneness and in [15] who fitted quasi Poisson regression model to U.K. own damage motor claim count data to take into account the possibility of within-cell heterogeneity.

In several research areas, negative binomial regression model has been suggested and applied for overdispersed count data. Recently, several parameterizations have been performed for the negative binomial regression model, and the two well known models, the NB-1 and the NB-2, have been proposed and applied [1-2][16-17][21]. However, the NB-1 and the NB-2 regression models are not nested and appropriate statistical tests to choose a better model cannot be carried out. Therefore, the functional form of negative binomial regression model has been extended and introduced as the NB-P model, where the NB-1 and the NB-2 models are special cases of the NB-P model when $P=1$ and $P=2$ respectively [23]. The motivation for the NB-P model is that the model can nests both the NB-1 and the NB-2 models parametrically and allows statistical tests of the two functional forms against a more general alternative. In particular, the likelihood ratio test can be implemented for choosing between NB-1 against NB-P, or NB-2 against NB-P. In addition to the negative binomial regression model, the generalized Poisson regression models such as the GP-1, the GP-2 and the GP-P have been proposed and applied for overdispersed as well as underdispersed count data [10][18-19][22].

This paper suggests the application of the two well known models of negative binomial regression, the NB-1 and the NB-2, and the functional form of negative binomial regression, the NB-P, for the analysis of vehicle theft crime data. This study contributes to the literatures of criminology through two elements; firstly, since little is known about the location and the descriptive nature of vehicle thefts in Malaysia, this study identifies and analyzes such factors, and hence, providing vital information to the Malaysian law enforcement agencies, car manufacturers and car owners for possible and practical steps of preventing, or at least limiting, the extent of vehicle thefts. Secondly, this study suggests the application of the two well known models of negative binomial regression, the NB-1 and the NB-2, and the functional form of negative binomial regression, the NB-P, for the analysis of vehicle theft crime. The advantage of using the NB-P model is that it parametrically nests the NB-1 and the NB-2 models, and therefore, allowing statistical tests of the NB-1 and the NB-2 models against a more general alternative.

\section{Material and Methods}

\subsection{Poisson Regression Model}

Let $\left(Y_{1}, Y_{2}, \ldots, Y_{2}\right)^{T}$ be the vector of count random variables and $n$ be the sample size. If $Y_{i}$ is distributed as Poisson, the probability mass function (p.m.f.) is,

$$
\operatorname{Pr}\left(Y_{i}=y_{i}\right)=\frac{\exp \left(-\mu_{i}\right) \mu_{i}^{y_{i}}}{y_{i} !}, y_{i}=0,1, \ldots
$$

with mean and variance $E\left(Y_{i}\right)=\operatorname{Var}\left(Y_{i}\right)$. To incorporate covariates and to ensure non-negativity, the mean or the 
fitted value is assumed to follow a $\log$ link, $E\left(Y_{i}\right)=\mu_{i}=\exp \left(x_{i}^{\prime} \beta\right)$, where $x_{i}$ denotes the vector of explanatory variables and $\beta$ the vector of regression parameters. Maximum likelihood estimates of $\beta$ can be obtained by maximizing the log likelihood.

\subsection{Functional Forms of Negative Binomial Regression Model}

The latent heterogeneity can be incorporated by rewriting the conditional mean of Poisson regression model as [12][21][23],

$$
\left.E\left(Y_{i} \mid \varepsilon_{i}\right)=\exp \left(x_{i}^{\prime} \beta \mid \varepsilon_{i}\right)\right)=k_{i} \mu_{i}
$$

where $k_{i}=\exp \left(\varepsilon_{i}\right)$ is assumed to follow gamma distribution with mean 1 and variance $\sigma^{-1}=a$,

$$
f\left(k_{i}\right)=\frac{v^{v} k_{i}^{v-1} \exp \left(-v k_{i}\right)}{\Gamma(v)}, k_{i}>0, v>0 .
$$

The conditional Poisson regression model is,

$$
\operatorname{Pr}\left(Y_{i}=y_{i} \mid k_{i}\right)=\frac{\exp \left(-k_{i} \mu_{i}\right)\left(k_{i} \mu_{i}\right)^{y_{i}}}{y_{i} !}, y_{i}=0,1, \ldots
$$

Using (2)-(3), the marginal is distributed as negative binomial with p.m.f.,

$$
\begin{aligned}
\operatorname{Pr}\left(Y_{i}=y_{i}\right) & =\int_{0}^{\infty} \frac{\mathrm{e}^{-k_{i} \mu_{i}}\left(k_{i} \mu_{i}\right)^{y_{i}}}{y_{i} !} \\
& -\frac{v^{v} k_{i}^{v-1} \exp \left(-v k_{i}\right)}{\Gamma(v)} \\
& \left.\left.=\frac{\Gamma\left(y_{i}+v\right)}{y_{i} ! \Gamma(v)}\left(\frac{v}{v+\mu_{i}}\right)^{v}\right)\left(\frac{\mu_{i}}{v+\mu_{i}}\right)^{y_{i}}\right), y_{i}=0,1, \ldots
\end{aligned}
$$

where the mean is $E\left(Y_{i}\right)=\mu_{i}$ and the variance is $\operatorname{Var}\left(Y_{i}\right)=$ $\mu_{i}\left(1+v^{-1} \mu_{i}\right)=\mu_{i}\left(1+a \mu_{i}\right)$. To incorporate covariates and to ensure non-negativity, the mean or the fitted value is assumed to follow a log link, $E\left(Y_{i}\right)=\mu_{i}=\exp \left(x_{i}^{\prime} \beta\right)$. The negative binomial regression model in (4) is also referred as the NB-2 [1].

Another parameterization for the negative binomial regression model is by letting $v=a^{-1} \mu_{i}$ in (4) to produce the NB-1, with mean $E\left(Y_{i}\right)=\mu_{i}$ and variance $\operatorname{Var}\left(Y_{i}\right)=\mu_{i}(1+a)$.

The NB-P regression model is produced by letting $v=$ $a^{-1} \mu_{i}^{2-P}$ in (4), so that the mean is $E\left(Y_{i}\right)=\mu_{i}$ and the variance is $\operatorname{Var}\left(Y_{i}\right)=\mu_{i}\left(1+a \mu_{i}^{P-1}\right)$, where $a$ denotes the dispersion parameter and $P$ the functional parameter [23].

The NB-1, the NB-2 and the NB-P regression models reduce to the Poisson regression model in the limit as $a \rightarrow 0$. If $a>0$, the variance exceeds the mean, and the NB-1, the NB-2 and the NB-P regression models allow overdispersion. In addition, the NB-P reduces to the NB-1 when $P=1$ and reduces to the NB-2 when $P=2$. Therefore, the NB-P parametrically nests both the NB-1 and the NB-2, and allows statistical tests of the two functional forms against a more general alternative.

Maximum likelihood estimates of $\beta$ and $a$ in NB-1 and NB-2 models can be obtained by maximizing their respective log likelihoods, and similarly, maximum likelihood estimates of $\beta, a$ and $P$ in NB-P model can be obtained by maximizing the NB-P log likelihood.

Table 1 summarizes the p.m.f., the mean and the variance of Poisson, NB-1, NB-2 and NB-P regression models. The advantage of understanding the mean-variance relationship of these regression models is that it allows the models to be compared in terms of mean-variance relationship. In particular, the mean-variance relationship of the NB-1 is in a linear form, the NB-2 in a quadratic form, and the NB-P in a functional form.

Table 1: P.m.f., mean and variance for Poisson and negative binomial models

\begin{tabular}{|l|l|l|}
\hline $\begin{array}{l}\text { Regression } \\
\text { model }\end{array}$ & P.m.f. & Mean and variance \\
\hline Poisson & $\frac{\mu_{i}^{i}}{y_{i} !} \exp \left(-\mu_{i}\right)$ & $\begin{array}{l}E\left(Y_{i}\right)=\mu_{i} \\
\operatorname{Var}\left(Y_{i}\right)=\mu_{i}\end{array}$ \\
\hline NB-1 & $\frac{\Gamma\left(y_{i}+\mu_{i} a^{-1}\right)}{y_{i} \Gamma\left(\mu_{i} a^{-1}\right)}\left(\frac{\mu_{i} a^{-1}}{\mu_{i} a^{-1}+\mu_{i}}\right)^{\mu_{i} a^{-1}}\left(\frac{\mu_{i}}{\mu_{i} a^{-1}+\mu_{i}}\right)^{y_{i}}$ & $\begin{array}{l}E\left(Y_{i}\right)=\mu_{i} \\
\operatorname{Var}\left(Y_{i}\right)=\mu_{i}(1+a)\end{array}$ \\
\hline NB-2 & $\frac{\Gamma\left(y_{i}+a^{-1}\right)}{y_{i} I \Gamma\left(a^{-1}\right)}\left(\frac{a^{-1}}{a^{-1}+\mu_{i}}\right)^{a^{-1}}\left(\frac{\mu_{i}}{a^{-1}+\mu_{i}}\right)^{y_{i}}$ & $\begin{array}{l}E\left(Y_{i}\right)=\mu_{i} \\
\operatorname{Var}\left(Y_{i}\right)=\mu_{i}\left(1+a \mu_{i}\right)\end{array}$ \\
\hline NB-P & $\frac{\Gamma\left(y_{i}+a^{-1} \mu_{i}^{2-P}\right)}{y_{i} ! \Gamma\left(a^{-1} \mu_{i}^{2-P}\right)}\left(\frac{a^{-1} \mu_{i}^{2-P}}{a^{-1} \mu_{i}^{2-p}+\mu_{i}}\right)^{a^{-1} \mu_{i}^{2-P}}\left(\frac{\mu_{i}}{a^{-1} \mu_{i}^{2-P}+\mu_{i}}\right)^{y_{i}}$ & $\begin{array}{l}E\left(Y_{i}\right)=\mu_{i} \\
\operatorname{Var}\left(Y_{i}\right)=\mu_{i}(1 \\
\left.a \mu_{i}^{P-1}\right)\end{array}$ \\
\hline
\end{tabular}

\subsection{Likelihood Ratio and Wald Tests}

Since the NB-1, the NB-2 and the NB-P regression models reduce to the Poisson regression model in the limit as $a \rightarrow 0$, the test of overdispersion in Poisson versus NB-1 and Poisson versus NB-2 models can be assessed by using likelihood ratio test. As an example, for testing Poisson against NB-1 regression models, the hypothesis is $H_{0}: a=0$ against $H_{0}: a>0$, which is a one-sided test, and the likelihood ratio is,

$$
T=2\left(\ln L_{1}-\ln L_{0}\right),
$$

where $\ln L_{1}$ and $\ln L_{0}$ denote the models' log likelihood under their respective hypothesis. Since the null hypothesis is on the boundary of parameter space, the likelihood ratio statistic is asymptotically distributed as half of probability mass at zero and half of chi-square with one degree of freedom [12]. The likelihood ratio in (5) can also be applied for testing the adequacy of NB-1 over NB-P and NB-2 over NB-P regression models, since the NB-P reduces to the NB-1 and the NB-2 when $P=1$ and $P=2$ respectively. As an example, for testing NB-1 
against NB-P, the hypothesis is $H_{0}: P=1$ against $H_{1}: P \neq 1$, which is a two-sided test, and the likelihood ratio statistic is asymptotically distributed as chi-square with one degree of freedom.

The test of overdispersion in Poisson against NB-1 and Poisson against NB-2 regression models can also be performed using Wald statistic, $\frac{\hat{a}^{2}}{\operatorname{Var}(\hat{a})}$. Since the null hypothesis is on the boundary of parameter space, the statistic is asymptotically distributed as half of probability mass at zero and half of chi-square with one degree of freedom. Similarly, the Wald statistic can be implemented for testing the adequacy of NB-1 over NB-P and NB-2 over NB-P regression models. The Wald statistic is $\frac{\hat{P}^{2}}{\operatorname{Var}(\hat{P})}$, where asymptotically, the statistic follows a chi-square with one degree of freedom.

\subsection{AIC and BIC}

When several maximum likelihood models are available, the performance of alternative models can be compared using several statistical criteria which have been proposed in statistical literatures. Two of the most regularly used measures are the Akaike Information Criteria (AIC) and the Bayesian Schwartz Information Criteria (BIC). The AIC penalizes a model with larger number of parameters and is defined asAIC $=-2 \ln L+2 p$, where $\ln L$ denotes the fitted $\log$ likelihood and $p$ the number of parameters. The BIC penalizes a model with a larger number of parameters and a larger sample size, and is defined as $\mathrm{BIC}=-2 \ln L+p \ln (n)$, where $n$ is the sample size. The best model is indicated by the smallest AIC and BIC.

\section{Results and Discussions}

In this study, crime data for private car theft obtained and compiled from ten insurance companies in Malaysia was considered. The data, which was based on 1.2 million private car policies for a three-year period of 2001-2003, was supplied by Insurance Services Malaysia (ISM). The exposure was expressed in terms of car-year unit, implying that the regression models were used for the analysis of vehicle theft crime where the rate was equal to the number of vehicle theft per car per year.

Table 2 shows the factors, or the covariates, which were considered for vehicle theft crime, represented by dummy variables. Initially, we have a total of $5 \times 5 \times 5 \times 5=625$ cross-classified claim counts to be fitted. However, by excluding zero exposure, we have a total of 557 count data to be fitted to Poisson, NB-1, NB-2 and NB-P regression models.

In this study, the NB-P regression model was fitted to vehicle theft crime data using $R$ program with $n l m$ function. For faster convergence, estimated parameters from fitting Poisson regression model were used as initial values. The NB-1 and the NB-2 regression models were also fitted using the same fitting procedure, by letting the functional parameter, $P$, to be fixed respectively at $P=1$ and $P=2$ for NB-1 and NB-2.

Table 3 shows the parameters, the log likelihood, the AIC and the BIC for the fitted models. The results indicate that the regression parameters for all models have similar estimates. As expected, the absolute value of $t$-ratios for NB-1, NB-2 and NB-P regression models are smaller than Poisson, indicating that the models' standard errors are larger than Poisson and the possible existence of overdispersion in the data.

For testing overdispersion in Poisson versus NB-1 regression models, $H_{0}: a=0$ vs. $H_{1}: a>0$, the likelihood ratio and the Wald statistics respectively are 538.86 and 7.40, indicating that the null hypothesis is rejected and the NB-1 is better than Poisson. Similarly, the likelihood ratio and the Wald statistics for testing overdispersion in Poisson versus NB-2 respectively are 565.24 and 7.18, also implying that the NB-2 is a better model.

For testing the adequacy of NB-1 versus NB-P regression models, $H_{0}: P=1$ vs. $H_{1}: P \neq 1$, and the adequacy of NB-2 versus NB-P regression models, $H_{0}: P=2$ vs. $H_{1}: P \neq 2$, the likelihood ratios are 52.28 and 25.90 respectively, and the Wald statistic is 18.59 , indicating that the NB-P is better than the NB-1 and the NB-2. The AIC and the BIC also imply that the NB-P is the best model compared to Poisson, NB-1 and NB-2 models.

Table 4 shows the parameters, the log likelihood, the $\mathrm{AIC}$ and the BIC for the NB-P regression model with covariates significant at $5 \%$ level. The regression parameters indicate that the risks of vehicle theft crime in Malaysia are higher for vehicles above 8 years (older car), vehicles above $1800 \mathrm{cc}$ (larger car), local vehicles (type $2)$, and vehicles located in the central area. 
Table 2: Covariates for vehicle theft crime data

\begin{tabular}{|l|l|}
\hline Factors & Classes \\
\hline Vehicle year & $0-1$ \\
\hline & $2-3$ \\
\hline & $4-5$ \\
\hline & $6-7$ \\
\hline & $8+$ \\
\hline Vehicle c.c. & $0-1000$ \\
\hline & $1001-1300$ \\
\hline & $1301-1500$ \\
\hline & $1501-1800$ \\
\hline & $1801+$ \\
\hline Vehicle make & Local type 1 \\
\hline & Local type 2 \\
\hline & Foreign type 1 \\
\hline & Foreign type 2 \\
\hline & Foreign type 3 \\
\hline Vehicle location & North \\
\hline & East \\
\hline & Central \\
\hline & South \\
\hline & East Malaysia \\
\hline
\end{tabular}

Table 3: Poisson, NB-1, NB-2 and NB-P regression models

\begin{tabular}{|c|c|c|c|c|c|c|c|c|}
\hline \multirow[t]{2}{*}{ Parameter } & \multicolumn{2}{|c|}{ Poisson } & \multicolumn{2}{|l|}{ NB-1 } & \multicolumn{2}{|l|}{ NB-2 } & \multicolumn{2}{|l|}{ NB-P } \\
\hline & Est. & $t$-ratio & Est. & $t$-ratio & Est. & $t$-ratio & Est. & $t$-ratio \\
\hline Intercept & -7.91 & -83.06 & -7.79 & -44.66 & -7.33 & -31.27 & -7.54 & -36.26 \\
\hline Year: 2-3 & 0.43 & 5.20 & 0.57 & 3.89 & 0.60 & 2.72 & 0.56 & 2.96 \\
\hline $4-5$ & 0.55 & 6.56 & 0.62 & 4.10 & 0.48 & 2.18 & 0.55 & 2.83 \\
\hline 6-7 & -0.17 & -1.79 & 0.00 & $\mid-0.01$ & -0.22 & -0.95 & -0.14 & $\mid-0.68$ \\
\hline $8+$ & 1.27 & 17.81 & 1.14 & 8.01 & 1.16 & 5.79 & 1.11 & 6.43 \\
\hline $\begin{array}{ll}\text { Cc: } & 1001- \\
1300 & \\
\end{array}$ & -0.41 & -5.62 & -0.38 & -2.96 & -0.46 & -2.21 & -0.46 & -2.71 \\
\hline $1501-1800$ & 1.00 & 16.32 & 0.92 & 7.72 & 0.46 & 2.54 & 0.68 & 4.24 \\
\hline $1801+$ & 1.66 & 26.30 & 1.51 & 12.17 & 1.36 & 7.70 & 1.45 & 9.48 \\
\hline $\begin{array}{l}\text { Make: } \\
\text { Foreign } \\
\text { type } 1\end{array}$ & -0.75 & -13.06 & -0.56 & -4.94 & -0.77 & -4.80 & -0.62 & -4.35 \\
\hline $\begin{array}{ll}\text { Foreign type } \\
2\end{array}$ & -0.78 & -9.15 & -0.59 & -3.63 & 0.04 & 0.19 & -0.33 & -1.81 \\
\hline $\begin{array}{ll}\text { Foreign } & \text { type } \\
3 & \end{array}$ & -2.55 & -16.93 & -2.33 & -8.57 & -1.55 & -4.87 & -2.01 & -6.62 \\
\hline $\begin{array}{l}\text { Location: } \\
\text { East }\end{array}$ & -0.28 & -1.81 & 0.00 & -0.02 & -0.59 & -2.34 & -0.34 & -1.29 \\
\hline Central & 1.33 & 18.35 & 1.16 & 9.20 & 1.21 & 6.68 & 1.17 & 7.62 \\
\hline South & 0.59 & 6.74 & 0.54 & 3.67 & 0.39 & 1.96 & 0.48 & 2.66 \\
\hline East Msia & -0.31 & -2.85 & -0.32 & -1.74 & -0.85 & -3.73 & -0.59 & -2.56 \\
\hline$a$ & - & - & 2.86 & 7.40 & 0.77 & 7.18 & 1.68 & 6.34 \\
\hline$P$ & - & - & - & - & - & - & 1.50 & 18.59 \\
\hline $\begin{array}{l}\text { Log } \\
\text { likelihood }\end{array}$ & \multicolumn{2}{|c|}{-1084.19} & \multicolumn{2}{|c|}{-814.76} & \multicolumn{2}{|c|}{-801.57} & \multicolumn{2}{|c|}{-788.62} \\
\hline AIC & \multicolumn{2}{|c|}{2198.38} & \multicolumn{2}{|c|}{1661.52} & \multicolumn{2}{|c|}{1635.13} & \multicolumn{2}{|c|}{1611.24} \\
\hline BIC & \multicolumn{2}{|c|}{2263.22} & \multicolumn{2}{|c|}{1730.68} & \multicolumn{2}{|c|}{1704.29} & \multicolumn{2}{|c|}{1684.72} \\
\hline
\end{tabular}

Table 4: NB-P regression model with significant covariates

\begin{tabular}{|l|l|l|}
\hline Parameter & Est. & $p$-value \\
\hline Intercept & -7.71 & 0.00 \\
\hline Year: $2-3$ & 0.62 & 0.00 \\
\hline $4-5$ & 0.62 & 0.00 \\
\hline $8+$ & 1.17 & 0.00 \\
\hline Cc: $1001-1300$ & -0.46 & 0.00 \\
\hline $1501-1800$ & 0.70 & 0.00 \\
\hline $1801+$ & 1.46 & 0.00 \\
\hline Make: Foreign type 1 & -0.62 & 0.00 \\
\hline Foreign type 3 & -0.35 & 0.03 \\
\hline Location: Central & 1.26 & 0.00 \\
\hline South & 0.57 & 0.00 \\
\hline East Msia & -0.48 & 0.01 \\
\hline$a$ & 1.75 & 0.00 \\
\hline$P$ & 1.48 & 0.00 \\
\hline Log likelihood & -789.69 \\
\hline AIC & 1609.39 \\
\hline BIC & 1674.23 \\
\hline
\end{tabular}

\section{Conclusion}

This paper proposes the application of the NB-1, the NB-2 and the NB-P regression models for the analysis of vehicle theft crime. The advantage of using the NB-P model is that it parametrically nests the NB-1 and the NB-2 models, and therefore, allowing statistical tests of the NB-1 and the NB-2 models against a more general alternative. In this study, the functional forms of negative binomial regression models were fitted to vehicle theft crime data obtained and compiled from ten insurance companies in Malaysia.

In Malaysia, vehicle theft contributes the majority of property crime, and the ratio of vehicle thefts to the overall theft has been consistent throughout the years. Therefore, it would definitely make a difference to the total crime index of the country if this particular crime is reduced. The difficult situation that the local motor industry encountered for such a long time is caused by several factors. The most formidable factors were those that lie outside the sector's control, which have the effect of significantly contributing to the escalating insurance claims continually experienced by the industry, such as the increasing number of motor vehicles on the road which resulted in a sharp increase in the number of vehicle theft and the number of insurance claims. On the other hand, several prevention and corrective measures can be implemented to reduce the number of stolen vehicles. As an example, manufacturers of motor vehicles may facilitate by making their vehicles more theft-proof by installing modern anti-theft devices such as anti-theft alarms and anti-theft key locks. Management of household and business properties may also assist by providing protected and secured parking, as well as hiring security guards. The Royal Malaysia Police may also 
strategize in increasing the number of police stations and locating them in strategic and "hot spot" areas, especially in the areas of high numbers of reported vehicle thefts. A study on the existence of anti-proof device and secured parking, as well as the number and the location of police stations in "hot spot" areas can be carried out if information on these factors is provided in police reports (for the number of stolen vehicle), or insurance claim reports (for the number of vehicle theft claim), or insurance policies (for the number of vehicle exposure). The information can be embedded in a regression model by using the contributing factors as covariates.

This study can be considered as a first step to investigate the link between vehicle theft crime with vehicle's characteristics and location. Based on the database of 1.2 million private car policies, the results indicate that the NB-P regression model is better than the Poisson, the NB-1 and the NB-2 regression models, and the regression parameters indicate that the risks of vehicle theft are higher for vehicles above eight years, vehicles above 1800 c.c., local vehicles and vehicles in the central area of Malaysia, where the capital of Malaysia, Kuala Lumpur, is located. The natural extension of this study is to apply the regression models to a wider range of data by merging reports of vehicle theft from Royal Malaysia Police (RMP), reports of motor insurance theft from Insurance Service Malaysia (ISM) and reports of socio-economic-demographic background from Department of Statistics Malaysia. Further investigation on the relationship between crime of vehicle theft with social, economic and demographic factors in Malaysia should be pursued. The relationship can be analyzed if the related and vital information such as population density, years of education, unemployment rates, youth population and immigrant rates for each district in Malaysia is available.

\section{Acknowledgements}

The research is financed by Fundamental Research Grant Scheme (Code: FRGS/1/2011/ST/UKM/02/8).

\section{References}

[1] A.C. Cameron, P.K. Trivedi. Econometric models based on count data: comparisons and applications of some estimators and tests. Journal of Applied Econometrics

[2] A.C. Cameron, P.K. Trivedi. Regression Analysis of Count Data, (1998). Cambridge University Press, New York.

[3] A.E. Renshaw. Modelling the claims process in the presence of covariates. ASTIN Bulletin 24 (1994) 265-285.

[4] A.S. Sidhu. The rise of crime in Malaysia: an academic and statistical analysis. Journal of the Kuala Lumpur Royal Malaysia Police College 4 (2005) 1-28.

[5] Department of Statistics Malaysia. Population distribution and basic demographic characteristic report 2010
Available: http://www.statistics.gov.my (November 18, 2011)

[6] G. Demombynes, B. Ozler. Crime and local inequality in South Africa. Journal of Development Economics 76 (2005) 265-292.

[7] G. Kleck, T. Chiricos.. Unemployment and property crime: a target-specific assessment of opportunity and motivation as mediating factors. Criminology 40 (2002) 649-680.

[8] General Insurance Association of Malaysia, 2011. Insurance industry statistics on stolen vehicles. Available: http://www.piam.org.my/news/piamnews/p014.htm ( June 22, 2011)

[9] Government Transformation Programme Roadmap. (2010). Chapter 6 - Reducing Crime. Available at http://portalintranet.kettha.gov.my/files/GTP\%20Roadmap.pdf (July 15, 2011)

[10] H. Zamani, N. Ismail. Functional form for the generalized Poisson regression model. Communications in Statistics (Theory and Methods) (in press) (2012) doi:10.1080/03610926.2011.564742.

[11] J.A. Nelder, Y. Lee. Likelihood, quasi-likelihood and pseudolikelihood: some comparisons. Journal of the Royal Statistical Society (B) 54 (1992) 273-284.

[12] J.F. Lawless. Negative binomial and mixed Poisson regression. Canadian Journal of Statistics 15 (1987) 209225.

[13] M. Aitkin, D. Anderson, B. Francis, J. Hinde.. Statistical modelling in GLIM. (1990) Oxford University Press, New York.

[14] M. Kelly. Inequality and crime. The Review of Economics and Statistics 82 (2000).530-539.

[15] M.J. Brockmann, T.S. Wright.. Statistical motor rating: making effective use of your data. Journal of the Institute of Actuaries 119 (1992) 457-543.

[16] N. Ismail, A.A. Jemain.. Handling overdispersion with negative binomial and generalized Poisson regression models. Casualty Actuarial Society Forum Winter (2007) 103-158.

[17] P. McCullagh, J.A. Nelder. Generalized Linear Models (2nd Edition) (1989) Chapman and Hall, London.

[18] P.C. Consul, F. Famoye. Generalized Poisson regression model. Communications in Statistics (Theory \& Methodology) 2 (1992) 89-109.

[19] P.C. Consul.. Generalized Poisson Distribution: Properties and Application. (1989) Marcel Dekker, New York.

[20] R. Di Tella, E. Schargrodsky.. Do police reduce crime? Estimates using the allocation of police forces after a terrorist attack. The American Economic Review 94 (2004) 115-133.

[21] R. Winkelmann Econometrics Analysis of Count Data (2008) Springer Verlag, Heidelberg.

[22] W. Wang, F. Famoye. Modeling household fertility decisions with generalized Poisson regression. Journal of Population Economics 10 (1997) 273-283.

[23] W. Greene. Functional forms for the negative binomial model for count data. Economics Letters 99 (2008) 585-590. 


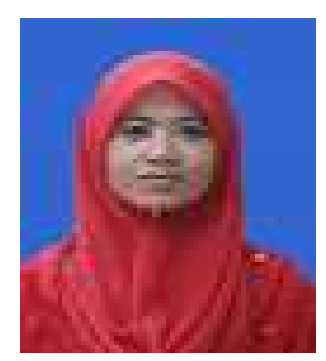

Malina
received
the MS degree in Applied
Statistics from University
Putra Malaysia in 2004,
and now she is doing her
PhD degree in Statistics from
the department of School
of Mathematics, Faculty
of Science and Technology,
University Kebangsaan

Malaysia.She is currently a Lecturer in University Utara Malaysia. Her research interests are in the areas of statistical modeling and survival analysis.

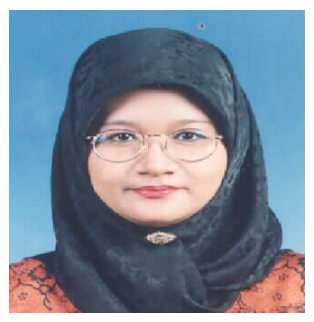

Noriszura
Ismail
in Actuarial Science
from University of Iowa
in 1993, and the PhD
degree in Statistics from
the University Kebangsaan
Malaysia in 2007. Now she is
one of the Associate Profesor
in University Kebangsaan
Malaysia. Her research
interests are in the areas of Actuarial Modeling and Statistical Modeling.

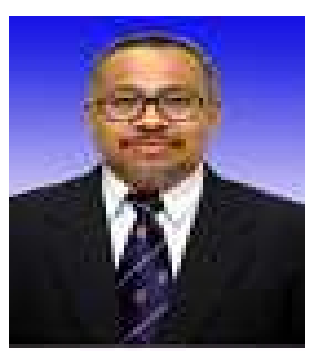

Ahmad Mahir Razali received the MS degree from University of Iowa, and the $\mathrm{PhD}$ degree from the Medical University of South Carolina. Now he is currently an Associate Profesor in University Kebangsaan Malaysia. His research interests are in the areas of environmental statistics, medical statistics, forecasting and benchmarking. 